\title{
Enteroparasitosis en menores de 11 años del Centro de Salud 9 de Enero- Chachapoyas. 2017
}

\section{Enteroparasitosis in children under 11 years of the Health Center January 9 - Chachapoyas. 2017}

\author{
Ernestina R. Vásquez Castro ${ }^{1}$
}

\begin{abstract}
RESUMEN
Los enteroparásitos intestinales son de importancia en salud pública en nuestro país, debido a que pueden producir complicaciones que comprometan la salud principalmente de la población infantil, por tal razón se planteó como objetivo determinar la prevalencia y características epidemiológicas de la enteroparasitosis en población menor de 11 años que acude al C.S 9 de Enero-Chachapoyas. Se trabajó con una población de 73 niños menores de 11 años, cuyas muestras fecales fueron analizadas mediante el método directo en solución fisiológica, solución de lugol, test de Graham y método de Ritchie. Los resultados registraron que la prevalencia de enteroparasitosis en los niños menores de 11 años fue de 60\%. Las enteroparasitosis fueron causadas mayormente por protozoarios en comparación con los helmintos. Siendo los protozoarios patógenos con mayor prevalencia: Blastocystis hominis (26\%), Giardia lamblia (21,9\%), y la prevalencia de helmintos fue de $(8,2 \%)$ Enterobius vermicularis. Las principales asociaciones entéricas fueron Entamoeba coli $(22,7 \%)$ en el caso de monoparasitismo, Blastocystis hominis y Entamoeba coli (11,4\%) en biparasitismo, no encontró asociación predominante para los casos de más de dos especies asociadas.
\end{abstract}

Palabras clave: Enteroparásitos, incidencia parasitaria.

\begin{abstract}
The intestinal enteroparasites are of importance in public health in our country, because they can produce complications that compromise the health of the infant population, for this reason the objective was to determine the prevalence and epidemiological characteristics of enteroparasitosis in population under 11 years that goes to the CS 9 de Enero-Chachapoyas. We worked with a population of 73 children under 11 years of age, whose faecal samples were analyzed by the direct method in physiological solution, Lugol's solution, Graham's test and Ritchie's method. The results showed that the prevalence of enteroparasitosis in children under 11 years old was $60 \%$. The enteroparasitosis were caused mostly by protozoa compared to helminths. The most prevalent pathogenic protozoa were: Blastocystis hominis (26\%), Giardia lamblia (21.9\%), and the prevalence of helminths was $(8.2 \%)$ Enterobius vermicularis. The main enteric associations were Entamoeba coli $(22.7 \%)$ in the case of monoparasitism, Blastocystis hominis and Entamoeba coli (11.4\%) in biparasitism, it did not find a predominant association for the cases of more than two associated species.
\end{abstract}

Keywords: Enteroparasites, parasitic incidence.

${ }^{1}$ Docente Asociada, Facultad de Educación y Ciencias de la Comunicación, Universidad Nacional Toribio Rodríguez de Mendoza de Amazonas 


\section{INTRODUCCIÓN}

Los enteroparásitos se encuentran ampliamente distribuidos en el mundo, teniendo mayor prevalencia en países en vías de desarrollo como Venezuela (Solano et al, 2008; Lemus-Espinoza et al, 2012), Paraguay (Echagüe et al, 2015), Argentina (Indelman et al, 2011), Brasil (Suescún, 2013). En nuestro país, uno de cada tres peruanos tiene más de un parásito (Borjas et al, 2009). La Dirección General de Epidemiología del Ministerio de Salud en el año 2012, consideró a la región Amazonas como una de las regiones con mayor índice de vulnerabilidad, precisando que entre los principales problemas de salud encontrados, según el análisis de las atenciones de consulta externa en los establecimientos del Ministerio de Salud, se encuentran las enfermedades infecciosas intestinales y helmintiasis (Valdez et al, 2013).

Las parasitosis intestinales son producidos principalmente por protozoos y helmintos, los cuales pueden ser patógenos o comensales (que producen escasa patología), (Indelman et al, 2011). Los parásitos intestinales son importantes en la salud porque repercuten en el estado nutricional del individuo privándolo de nutrientes (Solano et al, 2008), ocasionando anemia, deficiencia de vitamina A, retraso en el crecimiento, malnutrición, y trastornos del desarrollo físico y cognitivo (Echagüe et al., 2015).

Las parasitosis intestinales afectan mayormente a la población infantil de las áreas rurales y los barrios pobres marginalizados, siendo esa población susceptible de adquirirla, principalmente por su inmadurez inmunológica y escaso desarrollo de hábitos higiénicos, escasa cultura médica, deficiente saneamiento ambiental (Solano et al, 2008).

En el Perú se ha encontrado una alta prevalencia de parasitosis causadas por parásitos patógenos, así mismo las parasitosis intestinales constituyen un problema de salud pública principalmente en las regiones con escaso saneamiento ambiental, donde estos parásitos pueden ingresar al organismo por vía oral por los hábitos higiénicos y sanitarios deficientes que facilitan su transmisión y conservación; por otro lado, Suescún. (2013), refiere que las parasitosis intestinales pueden causar diversas manifestaciones clínicas como mala absorción, desnutrición, anemia, etc, dependiendo de la especie del parásito, evolución de la infección, estado inmunológico, nutricional entre otros factores socioeconómicos.

Dada las características ecológicas, sociales y sanitarias de la población del departamento de Amazonas, hace que reúnan condiciones propicias para el desarrollo de diversas enfermedades sobre todo aquellas causadas por parásitos intestinales protozoos y helmintos, sin embargo existen pocos reportes de estudios realizados en zonas urbanas como Chachapoyas, es por ello que el presente estudio investigó la prevalencia de parasitosis intestinal en niños menores de 11 años atendidos en el centro de salud 9 de Enero del Asentamiento Humano Santo Toribio de Mogrovejo, del distrito de Chachapoyas, siendo este tipo de estudio fundamental para tomar acciones de prevención y control de endoparasitosis, constituyendo una herramienta útil y rápida para orientar y monitorear las estrategias sanitarias que deben ser aplicadas y así mismo contribuir a mejorar la calidad de vida de la población más joven. En tal sentido, se tuvo como como objetivo determinar la prevalencia de parasitosis intestinal en niños menores de 11 años atendidos por consultorio médico del C.S 9 de EneroAAHH Santo Toribio de Mogrovejo-Chachapoyas

\section{MATERIAL Y MÉTODOS}

El presente trabajo de investigación, por sus características cualitativas, se enmarcó en un Diseño descriptivo de corte transversal. La población objeto de estudio fueron los niños menores de 11 años, atendidos durante los meses de abril - setiembre del año 2017 en consultorio médico del C.S 9 de Enero del Asentamiento Humano Toribio Rodríguez de Mendoza, distrito de Chachapoyas, provincia de Chachapoyas, Región Amazonas. La muestra estuvo constituida por 73 niños menores de 11 años que fueron atendidos en consulta médica durante los meses de abril a septiembre del 2017 en el C.S 9 de Enero -Chachapoyas. El tipo de muestreo fue no probabilístico por conveniencia y la recolección de muestras se realizó durante los meses de abril a setiembre de 2017, para lo cual los pacientes menores de 11 años de edad, atendidos por consultorio médico, con solicitud de examen parasitológico, fueron remitidos al laboratorio del centro de salud para recabar los envases de recolección.

Al padre, madre o tutor del paciente, se entregó un envase de plástico nuevo, limpio de boca ancha y tapa rosca, para la recolección de muestra de heces por deposición espontánea, además una lámina portaobjetos y cinta adhesiva para la toma del Test de Graham. Las muestras seriadas de heces fueron recepcionadas en el Laboratorio del C.S 9 de Enero, donde se realizó el examen parasitológico directo, posteriormente se añadió una solución conservante de formol $10 \%$ para ser transportadas en cajas de tecnopor al Laboratorio de Biología-UNTRM, para el análisis mediante el método de concentración.

Los criterios de inclusión fueron: niños de sexo masculino o femenino, menores de 11 años atendidos por consulta médica en el C. S 9 de Enero durante el 
período abril-setiembre 2017. Consentimiento informado firmado por el padre, madre o tutor del paciente de participar en el estudio de investigación.

El proyecto fue revisado por el Comité de Ética de la UNTRM, así mismo se contó con el consentimiento informado de los padres o tutores de los participantes a quienes se les informó sobre la naturaleza y objetivos del trabajo y así mismo fueron instruidos respecto a la recolección de la muestra de heces y la realización del test de Graham.

Se aplicó una encuesta estructurada a padres o tutores de los 73 niños que participaron en el estudio, que involucraba información socio-demográfica, síntomas clínicos, aspectos sanitarios, acceso al agua y desagüe y características de construcción de la vivienda y familiares. Estos datos incluyeron número de identificación, nombre, edad, sexo, residencia actual, características de la vivienda, servicio de agua y desagüe, servicio higiénico, presencia de animales domésticos y medicación antiparasitaria en los últimos 6 meses. Para el examen físico se incluyeron los datos: síntomas y signos, dolor abdominal, dispepsia a grasas, náuseas y vómitos, anorexia, astenia, coluria, diarrea, escalofríos, estreñimiento y prurito anal.

El análisis parasitológico consistió en colocar en una lámina portaobjetos una gota de solución salina fisiológica y luego se depositó una pequeña porción de heces con ayuda de un mondadientes, una vez emulsionada la muestra de heces, se puso la laminilla cubre objetos, observando las muestras en un microscopio compuesto en los objetivos de 10x y 40x, respectivamente.

El método directo con solución de lugol fue similar al procedimiento anterior, excepto que se utilizó una solución yodo-yodurada de lugol en lugar de la solución salina fisiológica.

El método de Graham, es un método específico para el diagnóstico de Enterobius vermicularis, consiste en adosar una cinta adhesiva en la zona perianal del paciente y luego montarla sobre una lámina portaobjetos para su observación a menor y mayor aumento en el microscopio para observar los huevos embrionados.

El método de concentración se utilizó el método de Ritchie (formol-éter), (Ritchie, 1948). Para detección de protozoarios, huevos o larvas de helmintos.

La información recabada en la ficha epidemiológica fue tabulada y se realizó un análisis univariado para la obtención de frecuencias y porcentajes con intervalos de confianza de $95 \%$ para las variables categóricas.

El análisis bivariado estableció las asociaciones entre parasitosis intestinal factores socio-ambientales y sanitarios asociados a la parasitosis investigados mediante el Chi-cuadrado para un nivel de significancia de $95 \%(\mathrm{p} \leq 0,05)$, valor establecido para aceptar la asociación entre variables.

\section{RESULTADOS}

Se realizó el análisis coproparasitológico de muestras procedentes de 73 niños menores de 11 años atendidos en consulta médica durante los meses de abril a setiembre del 2017 en el Centro de Salud 9 de Enero, de los cuales, el 52\% (38/72) pertenecieron al sexo masculino y el 48\% (35/73) al sexo femenino.

El porcentaje de muestras en las que se encontraron enteroparásitos fue el 60,3\% (44/73), en tanto que en el 39.7\% (29/73) no se hallaron parásitos. Así mismo, el 63,2\% (24/38) de los varones y el 57,1\% (20/35) de mujeres se encontraron parasitados. De acuerdo a la prueba de Chi-cuadrado con un $\mathrm{p} \leq 0,05$, al 95\% de confianza, no hubo relación entre sexo y parasitosis intestinal (Tabla 1 ).

Tabla 1. Parasitosis intestinal según sexo, en niños menores de 11 años del C.S. 9 de Enero, Santo Toribio de Mogrovejo-Chachapoyas, 2017

\begin{tabular}{|c|c|c|c|c|c|c|c|}
\hline \multirow{3}{*}{ Sexo } & \multicolumn{2}{|c|}{ Total } & & & & & \multirow{3}{*}{$\mathbf{P}$} \\
\hline & \multirow[t]{2}{*}{$\mathbf{N}$} & \multirow[t]{2}{*}{$\%$} & \multicolumn{2}{|c|}{ Parasitados } & \multicolumn{2}{|c|}{ No Parasitados } & \\
\hline & & & $\mathbf{N}$ & $\%$ & $\mathbf{N}$ & $\%$ & \\
\hline Masculino & 38 & 52 & 24 & 63.2 & 14 & 36.8 & \multirow{2}{*}{0,599} \\
\hline Femenino & 35 & 48 & 20 & 57.1 & 15 & 42.9 & \\
\hline Total & 73 & 100 & 44 & 60.3 & 29 & 39.7 & \\
\hline
\end{tabular}


De acuerdo al grupo etario, la mayor prevalencia de enteroparasitosis estuvo comprendido en niños del grupo etario de 1 a 3 años de edad, con 26\% (19/73), seguido del grupo etario de 4 a 6 años con 21,9\% (16/73), en tanto que la menor prevalencia de parasitosis intestinal se encontró en el grupo etario de niños menores a 1 año, $1,4 \%$ (1/73). Sin embargo, de acuerdo a la prueba Chi-cuadrado ( $\mathrm{p} \leq 0,05$, al 95\% de confianza), no hubo relación entre grupo etario y parasitosis intestinal (Tabla 2).

Tabla 2. Parasitosis intestinal según grupo etario en niños menores de 11 años del C.S. 9 de Enero, Santo Toribio de Mogrovejo -Chachapoyas, 2017

\begin{tabular}{lccccccc}
\hline \multirow{2}{*}{ Grupo Etario } & \multicolumn{2}{c}{ Muestras analizadas } & \multicolumn{2}{c}{ Parasitados } & \multicolumn{2}{c}{ No parasitados } & P \\
& Total & $\mathbf{\%}$ & Total & $\mathbf{\%}$ & Total & $\mathbf{\%}$ & \\
\hline Menor a 1 año & 4 & 5.5 & 1 & 1.4 & 3 & 4.1 & \\
1 - 3 años & 34 & 46.6 & 19 & 26.0 & 15 & 20.5 & \\
4 - 6 años & 22 & 30.1 & 16 & 21.9 & 6 & 8.2 & 0,4185 \\
7 - 9 años & 7 & 9.6 & 4 & 5.5 & 3 & 4.1 & \\
10 - 12 años & 6 & 8.2 & 4 & 5.5 & 2 & 2.7 & \\
\hline TOTAL & 73 & 100 & 44 & 60.3 & 29 & 39.7 & \\
\hline
\end{tabular}

Con respecto al grado de patogenicidad de los parásitos hallados en la población de niños estudiada, se observa que el $41,1 \%(30 / 73)$ fueron patógenos, mientras que el 19,2\% (14/73) correspondió al hallazgo de parásitos comensales o no patógenos (Tabla 3).

Tabla 3. Parasitosis intestinal según grado de patogenicidad de los parásitos, en niños menores de 11 años del C.S. 09 de Enero, Santo Toribio de Mogrovejo -Chachapoyas, 2017.

\begin{tabular}{lcc}
\hline \multicolumn{1}{c}{$\begin{array}{c}\text { Grado de } \\
\text { patogenicidad }\end{array}$} & N & \% \\
\hline Negativo & 29 & 39.7 \\
Parásitos patógenos & 30 & 41.1 \\
Parásitos no patógenos & 14 & 19.2 \\
\hline Total & 73 & 100 \\
\hline
\end{tabular}

La prevalencia de monoparasitismo en el total de niños evaluados fue de 30,1\% (22/73), mientras el $17,8 \%$ (13/73) presentó dos tipos de parásitos intestinales (biparasitismo) y el 12,3\% (9/73) poliparasitismo (3 o más especies parasitarias en co-infección) (Tabla 4).

Así mismo, en las muestras analizadas se hallaron siete especies distintas de parásitos patógenos y no patógenos o comensales, entre protozoarios y helmintos. Respecto a las especies patógenas, las más frecuentes fueron Blastocystis homnins (19/73) y Giardia lamblia, (16/73), ambos protozoarios, con prevalencia de $26 \%$ y $21,9 \%$ respectivamente; así mismo se registró Enterobius vermicularis, helminto cuya prevalencia fue de 8,2\% (6/73) de las muestras estudiadas. Las especies comensales no patógenas: Entamoeba coli (38,4\%,) tuvo mayor predominancia, seguido por Iodamoeba butschlii, Endolimax nana y Chilomastix mesnili con porcentajes menores al 6\% (Tabla 4$)$. 
Tabla 4. Prevalencia de parasitosis intestinal según tipo de infección, en niños menores de 11 años del C.S. 9 de Enero, Santo Toribio de Mogrovejo -Chachapoyas, 2017.

\begin{tabular}{|c|c|c|c|}
\hline \multirow{2}{*}{\multicolumn{2}{|c|}{ Tipo de infección }} & \multicolumn{2}{|c|}{ Prevalencia } \\
\hline & & \multirow{2}{*}{$\frac{\mathbf{N}}{29}$} & \multirow{2}{*}{$\frac{\%}{39.7}$} \\
\hline & Negativo & & \\
\hline & Monoparasitismo & 22 & 30.1 \\
\hline & Biparasitismo & 13 & 17.8 \\
\hline & Poliparasitismo & 9 & 12.3 \\
\hline & Parásitos patógenos & & \\
\hline & Protozoos & & \\
\hline \multirow[t]{3}{*}{ Protozoos } & Blastocystis hominis & 19 & 26.0 \\
\hline & Giardia lamblia & 16 & 21.9 \\
\hline & Helmintos & & \\
\hline \multirow[t]{3}{*}{ Helmintos } & Enterobius vermicularis & 6 & 8.2 \\
\hline & Parásitos no patógenos & & \\
\hline & Protozoos & & \\
\hline \multirow[t]{4}{*}{ Protozoos } & Entamoeba coli & 28 & 38.4 \\
\hline & Iodamoeba butschlii & 4 & 5.5 \\
\hline & Endolimax nana & 3 & 4.1 \\
\hline & Chilomastix mesnilii & 1 & 1.4 \\
\hline
\end{tabular}

Según el tipo de parasitismo y grupo etario, se observa que la población parasitada, el grupo etario con mayor prevalencia de monoparasitismo fue el de 1 a 3 años seguido del de 4 a 6 años de edad con 25\% (11/44) y 18,2\% (8/44),respectivamente. Similar tendencia se observó para el caso de biparasitismo, con prevalencias de 13,6\% (6/44) para el grupo etario de 1 a 3 años y 9,1\% (9/44), para el grupo etario de 4 a 6 años de edad. En tanto que el grupo etario que registró mayor porcentaje de poliparasitismo fue el de 4 a 6 años, con 9, 1\% (4/44), (Tabla 5).

Tabla 5. Parasitismo intestinal en niños menores de 11 años del C.S. 09 de Enero, Santo Toribio de Mogrovejo Chachapoyas, 2017, según tipo de parasitismo y grupo etario.

\begin{tabular}{lccccccc}
\hline & \multicolumn{2}{c}{ Enteroparasitosis } & \multicolumn{5}{c}{ Grupo Etario } \\
Asociación parasitaria & $\mathbf{N}$ & $\mathbf{\%}$ & Menor a 1 año & $\mathbf{1} \mathbf{- 3}$ años & $\mathbf{4}-\mathbf{6}$ años & $\mathbf{7} \mathbf{- 9}$ años 10 - 12 años \\
\hline Monoparasitismo & 22 & 30.1 & 0 & 11 & 8 & 2 & 1 \\
Biparasitismo & 13 & 17.8 & 1 & 6 & 4 & 0 & 2 \\
Poliparasitismo & 9 & 12.3 & 0 & 2 & 4 & 2 & 1 \\
\hline Total & 44 & 60.2 & 1 & 19 & 16 & 4 & 4 \\
\hline
\end{tabular}

Teniendo en cuenta la asociación parasitaria según el tipo de parasitismo, se puede observar que del total de casos de monoparasitismo, la especie patógena más frecuente fue $B$. hominis 13,6\% (6/44), en tanto que E. coli, parásito comensal de mayor prevalencia, fue 22,7\% (10/44). En casos de biparasitismo, las asociaciones predominantes fueron B.hominis - E. coli y G.lamblia - E. coli, con $11,4 \%$ y y $9,1 \%$ de los casos positivos respectivamente.

En relación al poliparasitismo, se puede observar que hubo asociación hasta de 4 especies parasitarias, en las cuales E. coli estuvo presente en la mayoría de los casos (15,8\%), (Tabla 6).

Tabla 6. Asociación parasitaria frecuente según tipo de parasitismo en niños menores de 11 años del C.S. 09 de Enero, Santo Toribio de Mogrovejo -Chachapoyas, 2017. 


\begin{tabular}{lcc}
\hline Especie/asociación & N & $\%$ \\
\hline Monoparasitismo & 4 & 9.1 \\
Giardia lamblia & 10 & 22.7 \\
Entamoeba coli & 6 & 13.6 \\
Blastocystis hominis & 1 & 2.3 \\
Endolimax nana & 1 & 2.3 \\
Chilomastix mesnilii & & \\
Biparasitismo & 4 & 9.1 \\
G. lamblia, E. coli & 1 & 2.3 \\
G. lamblia, B. hominis & 5 & 11.4 \\
B. hominis, E. coli & 1 & 2.3 \\
E. nana, B. hominis & 2 & 4.5 \\
E. coli, Enterobius vermicularis & & \\
Poliparasitismo & 2 & 4.5 \\
G.lamblia, E. coli, B. hominis & 1 & 2.3 \\
G. lamblia, B. hominis, E.vermicularis & 2 & 4.5 \\
G.lamblia. I. butschlii, E.coli & 2 & 4.5 \\
B. hominis, E. coli, E. vermiculatis & 1 & 2.3 \\
G.lamblia., I. butschlii,B. hominis, E.coli & 2.3 \\
G.lamblia. Iodamoeba butschlii,Endolimax nana, E. vermicularis 1 & 44 & 100.0 \\
\hline Total & &
\end{tabular}

El análisis bivariado empleando la prueba de Chi-cuadrado con un $p \leq 0,05$, valores mayores a éste nos indicó que no hubo dependencia entre las variables sociodemográficas estudiadas: sexo, lugar de nacimiento, dirección actual y viaje reciente, con el parasitismo intestinal en niños menores de 11 del C.S. 09 de Enero, Santo Toribio de Mogrovejo -Chachapoyas, 2017, (Tabla 7).

Tabla 7. Parasitosis intestinal según factores sociodemográficos en niños menores de 11 años del C.S. 09 de Enero, Santo Toribio de Mogrovejo-Chachapoyas, 2017.

\begin{tabular}{|c|c|c|c|c|c|}
\hline \multirow{3}{*}{ Variables } & \multirow{2}{*}{\multicolumn{2}{|c|}{ Total de niños }} & \multicolumn{2}{|c|}{ Niños } & \multirow{3}{*}{$\mathbf{P}$} \\
\hline & & & & dos & \\
\hline & $\mathbf{N}$ & $\%$ & $\mathbf{N}^{\circ}$ & $\%$ & \\
\hline \multicolumn{6}{|l|}{ Sexo } \\
\hline Masculino & 38 & 52.1 & 24 & 54.5 & \multirow[t]{2}{*}{0,5998} \\
\hline Femenino & 35 & 47.9 & 20 & 45.5 & \\
\hline \multicolumn{6}{|c|}{ Lugar de nacimiento } \\
\hline Chachapoyas & 59 & 80.8 & 34 & 77.3 & \multirow[t]{3}{*}{0,5659} \\
\hline Otro distrito & 9 & 12.3 & 6 & 13.6 & \\
\hline Fuera de Amazonas & 5 & 6.8 & 4 & 9.1 & \\
\hline \multicolumn{6}{|l|}{ Dirección actual } \\
\hline \multicolumn{2}{|c|}{ Santo Toribio de Mogrovejol } & 69.9 & 30 & 68.2 & \multirow[t]{3}{*}{0,8523} \\
\hline Otro barrio & 20 & 27.4 & 13 & 29.5 & \\
\hline fuera de Chacha & 2 & 2.7 & 1 & 2.3 & \\
\hline \multicolumn{6}{|c|}{ Viajó recientemente } \\
\hline No viajó & 67 & 91.8 & 41 & 93.2 & \multirow[t]{2}{*}{0,5914} \\
\hline sí viajó & 6 & 8.2 & 3 & 6.8 & \\
\hline
\end{tabular}

La asociación de las manifestaciones clínicas y parasitismo intestinal en la población de niños estudiada, se encontraron diferencias significativas de acuerdo a la prueba Chi-cuadradoen las variables dificultad al defecar y 
estreñimiento, lo que demuestra la dependencia de estas variables con los casos de enteroparasitismo (Tabla 5).

Para los demás datos clínicos investigados no se encontraron diferencias significativas de acuerdo a la prueba Chicuadrado, (Tabla 8).

Tabla 8. Parasitosis intestinal según datos clínicos en niños menores de 11 años del C.S. 09 de Enero, Santo Toribio de Mogrovejo-Chachapoyas, 2017.

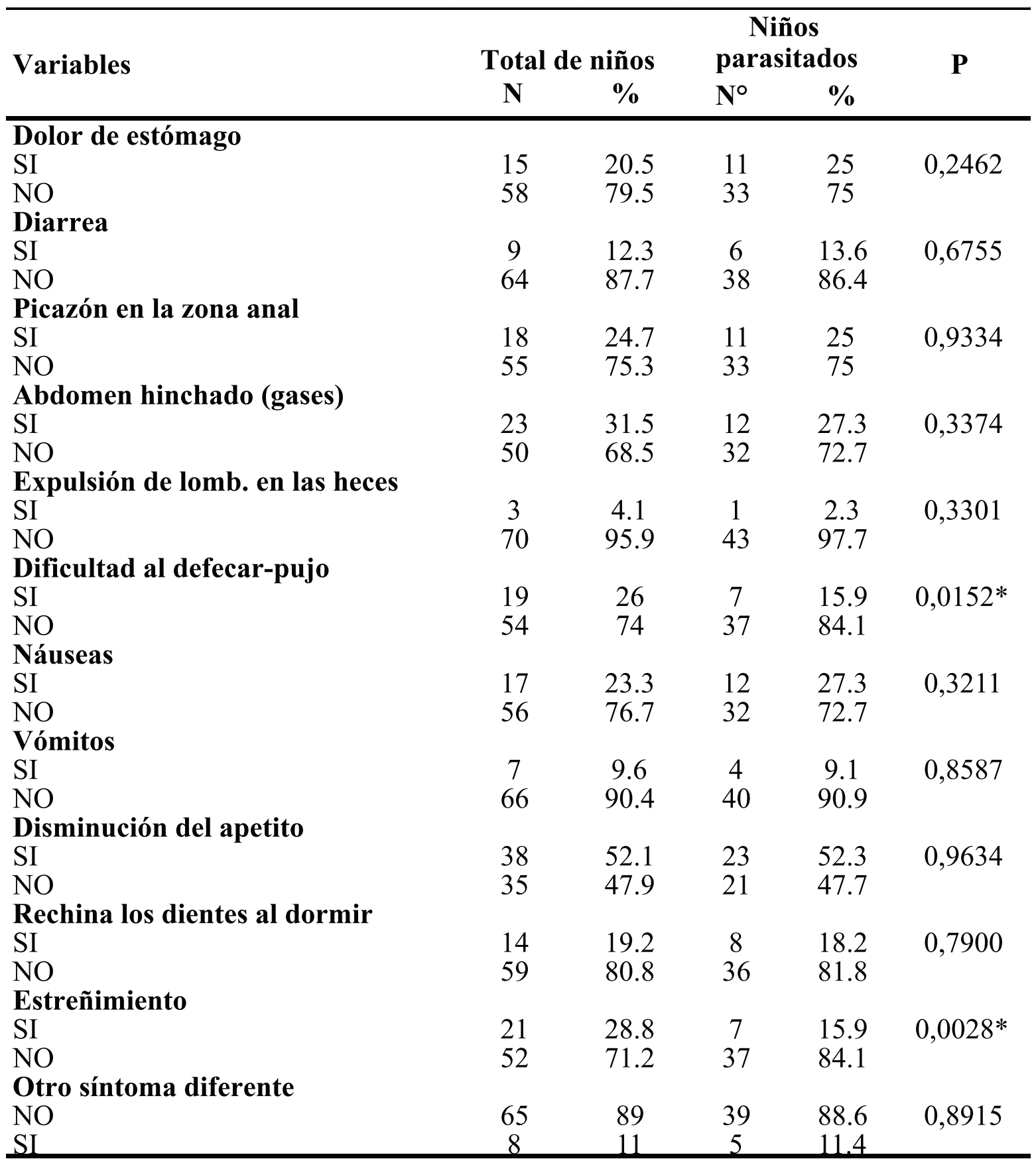

*relación estadísticamente significativa con el parasitismo intestinal $p \leq 0,05$, al 95\% de confianza. 
Las características socioeconómicas-ambientales relacionadas con la vivienda de los niños menores de 11 años evaluados en el presente estudio, revelan que un porcentaje alto de niños parasitados, presentan viviendas de adobe $(69,9 \%)$, con piso de cemento (33\%), tienen acceso al suministro de agua potable $(93,2 \%)$, sistema de desagüe $(84,9 \%)$, cuentan con baño propio $(82,2 \%)$, así como eliminación de la basura a través del carro recolector (94,5\%). Así como el 69,9\% poseen animales domésticos en la vivienda; sin embargo, la prueba de Chi-cuadrado reveló dependencia entre la variable tipo de piso de la vivienda con la parasitosis intestinal ( $\mathrm{p} \leq 0,05$, al 95\% de confianza), (Tabla 9).

Tabla 9. Parasitosis intestinal según Factores socioeconómicos ambientales relacionados con la vivienda, en niños menores de 11 años del C.S. 09 de Enero, Santo Toribio de Mogrovejo-Chachapoyas, 2017.

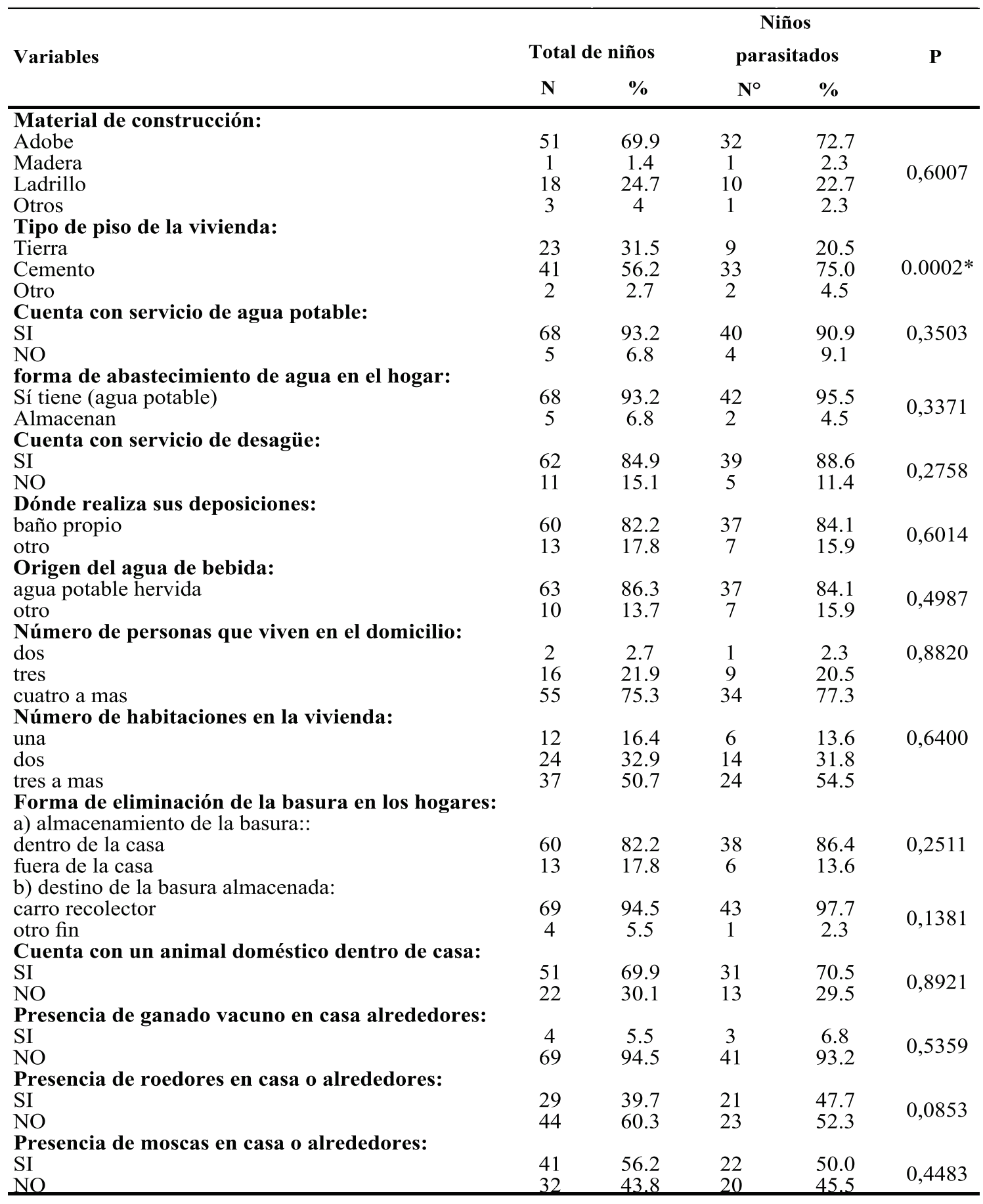

*relación estadísticamente significativa con el parasitismo intestinal p $\leq 0,05$ al 95\% de confianza. 
En todos los casos el análisis estadístico no registró diferencias significativas de acuerdo a la prueba Chi-cuadrado en las variables estudiadas, $(\mathrm{p} \leq 0,05$, al 95\% de confianza) (Tabla 10).

Tabla 10. Parasitosis intestinal según Factores socioeconómicos ambientales relacionados con el paciente, en niños menores de 11 años del C.S. 09 de Enero, Santo Toribio de Mogrovejo-Chachapoyas, 2017.

${ }^{1}$ ns: no significativo de acuerdo a la prueba $\chi^{2} \mathrm{p} \leq 0,05$ al $95 \%$ de confianza.

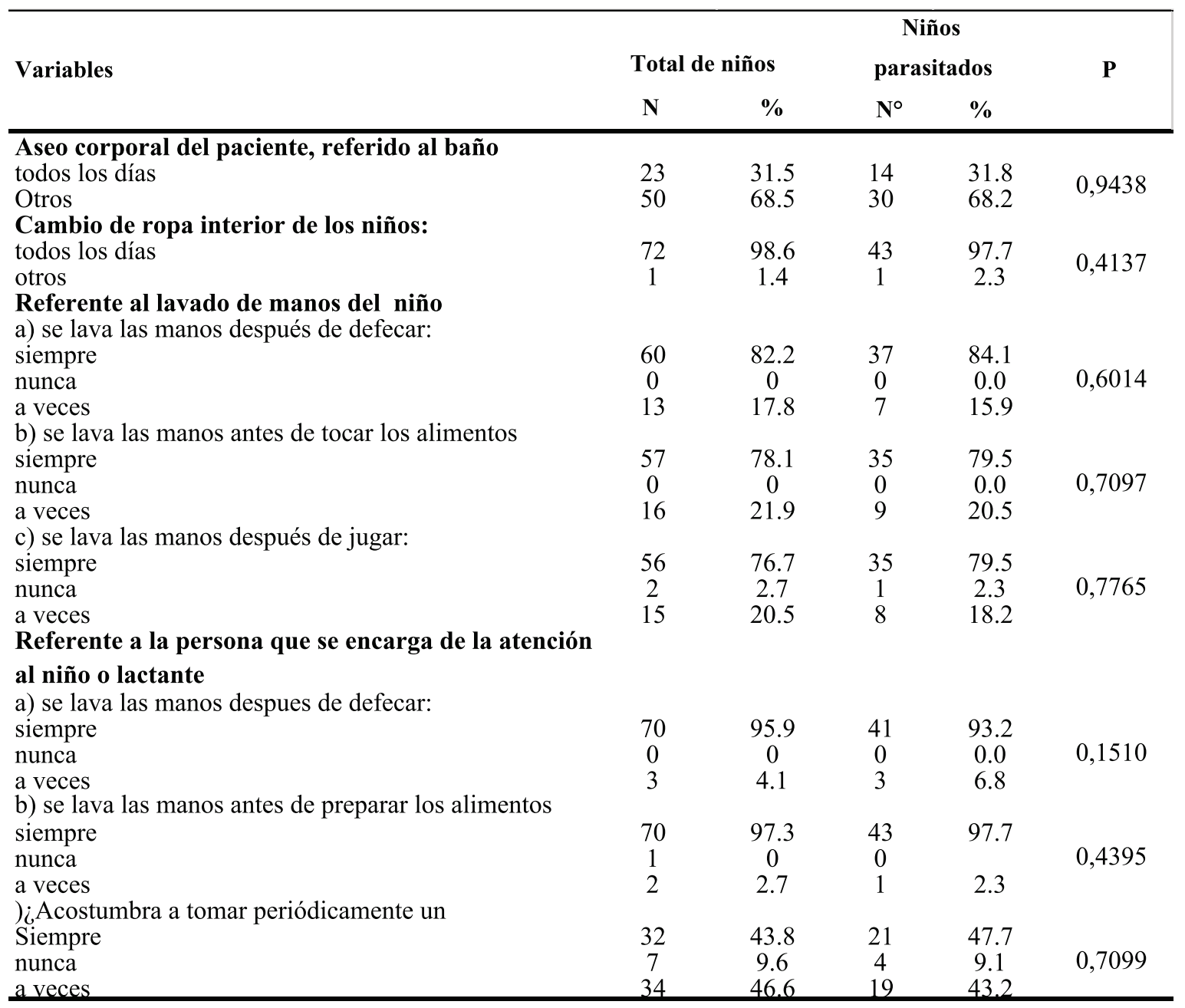

\section{DISCUSIÓN}

Las parasitosis intestinales representan un problema de salud pública en diferentes lugares del mundo, afectando principalmente a la población infantil. Son pocos los estudios realizados sobre parasitosis en la ciudad de Chachapoyas, por cuanto los resultados serán discutidos con los trabajos realizados en otras ciudades del Perú y del mundo.

En el presente estudio se determinó la prevalencia de enteroparasitosis en el 60,3\% de niños menores de 11 años, quienes provenían del AA.HH. Santo Toribio de Mogrovejo, distrito de Chachapoyas. Reportes con mayor prevalencia de parasitosis intestinal han sido registrados en Caquetá- Colombia 90\%, (Lucero, 2015); La PlataArgentina 81,3\%, (Zonta et al, 2013); Anzoátegui-Venezuela 74,6\% (Lemus et al, 2012). En el Perú,v Valladares (2016) reportó el 85,3\% de enteroparasitosis en niños de 8 a 13 años de edad, del distrito de San Juan de Miraflores, Lima. Morales, (2016) registró en 90,6\% la parasitosis de niños en edad pre escolar de un centro médico de Essalud- Celendín, Cajamarca, sin embargo resultados de prevalencia de parasitosis, similares al presente estudio fueron reportados por Rivera, (2015) en Leimebamba-Amazonas y en Andahuaylas por Altamirano, (2014) con porcentajes de 64,2\% y $42 \%$, respectivamente. 
La menor prevalencia de niños parasitados de parasitados fue hallado en el grupo etario de niños menores de 1 año de edad, valor que coincide con el reporte de otros investigadores como Lemus, (2012), lo cual podría estar relacionado a los buenos hábitos de higiene en los cuidados maternos, así como una mínima exposición a factores de riesgo en esta edad.

No hubo dependencia entre las variables sexo y el parasitismo intestinal en la población estudiada, así mismo se encontró un predominio de monoparasitismo, resultados similares reportó Echagüe et al, (2015) en niños menores de 5 años de comunidades rurales de Paraguay; Mera et al, (2015), en un estudio realizado sobre la distribución de las enteroparasitosis en un pueblo joven de Lambayeque, tampoco encontró asociación sociodemográfica y parasitosis intestinal entre la presencia de estar infectado al menos por un parásito, la edad, el sexo, condiciones de saneamiento y vivienda.

La baja prevalencia de poliparasitismo en los niños menores de 11 años que participaron en el

presente estudio podría responder a la políticas de prevención y desparasitación iniciada por el Gobierno Central, quien declaró "Día de la Desparasitación" a partir del año 2018, el tercer domingo de los meses de marzo y de setiembre de cada año; y, excepcionalmente el 9 de julio para el año 2017 mediante Resolución Ministerial N ${ }^{\circ}$ o 4472017/MINSA.

En el estudio realizado, los niños parasitados evidencian notoriamente el predominio de protozoos en relación a helmintos, Respecto a las especies patógenas, las más frecuentes fueron Blastocystis homnins (26\%) y Giardia lamblia, (21.9\%), ambos protozoarios y de las especies comensales no patógenas, Entamoeba coli (38.4\%,) tuvo mayor predominancia, seguido por Iodamoeba butschlii, Endolimax nana y Chilomastix mesnili en porcentajes menores a $6 \%$. Resultados similares fueron hallados por Valladares, (2016); Rivera, (2015); Morales, (2016) y Navarro, (2013) debido probablemente a que las condiciones del clima y calidad sanitaria del agua, determinan mayor prevalencia de protozoarios en la costa y sierra y de helmintos en la selva.

Enterobius vermicularis fue el único helminto encontrado en el estudio con una prevalencia de $8,2 \%$ en los niños parasitados; así mismo, Zevallos (citado por Valladares, 2016), también reportó una menor prevalencia de este helminto, (15\%), empleando el mismo método de cinta engomada, estos menores porcentajes pueden deberse a los adecuados hábitos de higiene y condiciones sanitarias de la población; otras causas atribuibles pueden ser la inadecuada aplicación de la técnica de toma de muestra del tutor o familiar responsable.

La mayoría de los hogares de pacientes parasitados del presente estudio cuentan con suministro de agua potable y recolección de residuos domiciliaros, similar a lo reportado por Zonta et al. (2013) en La Plata-Argentina; Rocha. (2003) en Colombia; Valladares. (2006) en Lima, quienes reportaron prevalencias de parasitosis intestinal mayores al $60 \%$, concordando en que no hubo dependencia entres estas variables y los casos de parasitosis intestinal, como lo señalado en el presente estudio. Así mismo Fuentes et al. (2007), al igual que en nuestro estudio tampoco encontró dependencia entre parasitosis intestinal y síntomas gastrointestinales, disposición de excretas, presencia de vectores, antecedentes de tratamiento antiparasitario, entre otros.

\section{CONCLUSIONES}

La prevalencia de enteroparasitosis en los niños menores de 11 años atendidos en consultorio médico en el C.S 9 de Enero del C.S. 09 de Enero, Santo Toribio de Mogrovejo-Chachapoyas fue de $60 \%$.

Las enteroparasitosis fueron causadas mayormente por protozoarios en comparación con los helmintos. Siendo los protozoarios patógenos con mayor prevalencia: Blastocystis hominis (26\%), Giardia lamblia $(21,9 \%)$, y la prevalencia de helmintos fue de $(8,2 \%)$ Enterobius vermicularis.

Las principales asociaciones entéricas fueron Entamoeba coli $(22,7 \%)$ en el caso de monoparasitismo, Blastocystis hominis y Entamoeba coli $(11,4 \%)$ en biparasitismo, no encontró asociación predominante para los casos de más de dos especies asociadas.

Los síntomas clínicos asociados a la parasitosis intestinal fueron dificultad al defecar o pujo y el estreñimiento.

El factor socioeconómico tipo de piso de vivienda estuvo asociado a la parasitosis intestinal.

Los factores sociodemográficos tales como edad, sexo, procedencia, y los factores socioeconómicos: servicio de agua potable, desagüe, eliminación de basura, presencia de vectores y hábitos de higiene, no tuvieron dependencia con los casos de parasitosis intestinal.

\section{REFERENCIAS BIBLIOGRÁFICAS}

Altamirano Zevallos, F. V. (2017). Factores de riesgo asociados a parasitismo intestinal en niños pre 
escolares atendidos en el Aclas San Jerónimo. Andahuaylas-2014.

Cordón, G. P. (2007). Formación de escuelas saludables: estudio de parásitos intestinales en niños de la provincia de Trujillo (Perú): tesis doctoral (Doctoral dissertation, Editorial de la Universidad de Granada).

Claros, A. M., Peña, M. M., López, M. G., Pérez, R. P., \& Fontelos, P. M. (2012). Parasitosis intestinales. Protocolos diagnóstico-terapéuticos de la AEP: Infectología pediátrica, 77-88.

Borjas, P., Arenas, F. \& Angulo-Bazán, Y. (2009). Enteroparasitismo en niños y su relación con la pobreza y estado nutricional. Ciencia $e$ Investigación Médica Estudiantil Latinoamericana, 14(1): 49-54.

Carmona-Fonseca, J., Peñuela, U., Rosa, M., \& Correa Botero, A. M. (2009). Parasitosis intestinal en niños de zonas palúdicas de Antioquia(Colombia). Iatreia, 22(1), 27-46.

Echagüe, G., Sosa, L., Díaz, V., Ruiz, I., Rivas, L., Granado, D., Funes, P., Zenteno, J., Pistilli, N., \& Ramírez, M. (2015). Enteroparasitosis en niños bajo 5 años de edad, indígenas y no indígenas, de comunidades rurales del Paraguay. Revista Chilena de Infectología, 32(6): 649-657.

Hernández Sampieri, R., Fernández Collado, C., \& Baptista Lucio, P. (1998). Metodología de la investigación. México: Editorial Mc Graw Hill, $15-40$.

Indelman, P.C., Echenique, C., Bertorini, G., Racca, L., Gómez, C., Luque, A. \& Magaró, H.M. (2011). Parasitosis intestinales en una población pediátrica de la ciudad de Rosario, Santa Fe, Argentina. Acta Bioquímica Latinoamericana 45(2): 329-334.

Lemus-Espinoza, D., Maniscalchi, M.T., Kiriakos, D., Pacheco, F., Aponte, C., Villarroel, O., Harb, P. \& García, O. (2012). Enteroparasitosis en niños menores de 12 años del estado Anzoátegui, Venezuela. Revista de la Sociedad Venezolana de Microbiología 32: 139-147.

Lucero-Garzón, T. A., Alvarez-Mota, L. A., Chicue, J. F., López, D., \& Mendoza, C. A. (2015). Parasitosis intestinal y factores de riesgo en niños de los asentamientos subnormales, FlorenciaCaquetá, Colombia. Facultad Nacional de Salud Pública, 33(2), 171-180.

Marcos, L.A., Maco, V., Terashima, A., Samalvides, F \&. Gotuzzo, E. (2002). Prevalencia de parasitosis intestinal en niños del valle del Mantaro, Jauja, Perú. Revista Médica Herediana 13: 85-90.

Mera-Olivares, A., Ganoza-Granados, L., Arce-Gil, Z., Alarcón-Benavides, E., Moreno-Echendía, G. M., \& Jiménez, F. L. (2015). Distribución de las enteroparasitosis en un pueblo joven de Lambayeque. Rev. Cuerpo Med. HNAAA, 6(3), 22-27.

Morales Del Pino, J. R. (2016). Parasitosis intestinal en preescolares y escolares atendidos en el centro médico EsSalud de Celendín, Cajamarca. Horizonte médico, 16(3), 35-42.

Navarro, M. (2013). Prevalencia de parasitosis intestinal y factores epidemiológicos asociados en escolares del asentamiento humano Aurora Díaz de Salaverry-Trujillo.

Núñez Zúñiga, A. L., \& Romero Buenaño, F. J. (2011). Incidencia de la parasitosis en los niños de la escuela fiscal mixta "general julio andrade", previa cloración agua de la parroquia de ilapo, cantón guano, provincia de chimborazo, en el periodo 1 de (Bachelor's thesis, Riobamba: Universidad Nacional de Chimborazo, (2011)).

Pascual, G., Iannacorie, J., Hernández, A. \& Salazar, N. (2010). Parásitos intestinales en pobladores de dos localidades de Yurimaguas, Alto Amazonas, Loreto, Perú. Neotropical Helminthology 4(2): 127-136.

Sajamí, P., \& Carola, K. (2017). Factores relacionados con parasitosis intestinal y su prevalencia en infantes de 0 a 5 años, atendidos en los meses de febrero a julio, en el C.S Moronacocha. Iquitos-Perú, 2014.

Salazar, C. R. (2017). Frecuencia y aspectos epidemiológicos del parasitismo intestinal en estudiantes de 5 a 16 años de Leimebamba, Amazonas-Perú. Revista Científica Pakamuros, 3(1), 6 .

Solano, L., Acuña, I., Barón, M; Morón, A. \& Sánchez, A. (2008). Influencia de la parasitosis intestinales y otros antecedentes infecciosos sobre el estado nutricional antropométrico de niños de situación de pobreza. Parasitología Latinoamericana 63: 12-19.

Tassara O., Renzo. (1999). Enteroparasitosis: realidad actual y manejo. Revista chilena de pediatría, 70(5), 441-445. 
Valdez, W., Napanga, E.O., Oyola, A., Mariños, J.C., Vílchez, A., Medina, J., \& Berto, M. (2013). Análisis de situación de salud del Perú. In Análisis de situación de salud del Perú (pp. 136).

Valladares, J.A. (2016). Prevalencia de enteroparásitos en niños de 8 a 13 años de edad de la Institución educativa $N^{\circ} 6041$ "Alfonso Ugarte" del distrito de San Juan de Miraflores". (Tesis de Pregrado), Universidad Ricardo Palma, Lima, Perú.

Zonta, M. L., Bergel, M. L., Cociancic, P., Gamboa, M. I., Garraza, M., Cesani, M. F., \& Navone, G. T. (2013). Enteroparasitosis en niños de Villaguay, Entre Ríos: un estudio integrado al estado nutricional y al ambiente. Rev Arg Parasitol, 1(2), 86-109. 